\title{
The reconfiguration of task set has no effect on the efficiency of feature search
}

\author{
Tao Gao, Mowei Shen, Rende Shui, and Zaifeng Gao \\ Zhejiang University, Hangzhou, China
}

\begin{abstract}
In this study, we investigated whether or not the reconfiguration of a set task could modulate the efficiency of feature search. In Experiment 1, target set size was manipulated. The results revealed that enumerating targets defined by color yields a much steeper slope when executed simultaneously with a discrimination task than when done singly. However, when distractor set size was manipulated, there was no significant difference in the efficiency of individualizing targets from distractors between the two task conditions, no matter whether the search was guided by bottom-up information (Experiment 2) or top-down information (Experiment 3). Drawing upon these results, the authors propose that (1) an additional task can impair the efficiency of enumeration, resulting in a steep slope of counting even when target set size is quite small, and (2) in feature search, the visual system first reconfigures the task set to match the input feature information and then detects the targets with an optimal efficiency, which does not support the theory proposed by Di Lollo, Kawahara, Zuvic, and Visser (2001; Di Lollo, Smilek, Kawahara, \& Ghorashi, 2005).
\end{abstract}

Search for a particular feature is highly efficient (Treisman \& Gelade, 1980), and with a simple glimpse of a scene, a target feature can pop out from distractors (Hochstein \& Ahissar, 2002). To what extent can this efficient search (defined as producing a nearly flat slope for the performance $\times$ set size function) be modulated by top-down control? Previous research (Müller, Reimann, \& Krummenacher, 2003; Wolfe, Butcher, Lee, \& Hyle, 2003) has revealed that the overall performance of a feature search can be impaired when the target feature and the task set do not match. However, whether an unsuitable task set can drastically increase the slope of a feature search is still controversial.

In Müller and colleagues' research (Müller et al., 2003), prior to the feature search task, observers were precued to the most probable target-defining dimension on a given trial. The results revealed expedited reaction times (RTs) for valid dimension targets, relative to neutral cue conditions, and lengthened RTs for invalid dimension targets. Another recent study (Wolfe et al., 2003) showed that in a singleton detection task, when the target-defining dimension was uncertain (making it impossible for observers to set an explicit top-down control), there was a cost in RTs, in comparison with when the target-defining dimension was fixed. In light of these results, Wolfe et al. (2003) argued that top-down control can modulate parallel processing by increasing the weight for a specific dimension in the activation map that sums up activations from all feature dimensions. If the target appears in that dimension, the salience of difference between the target and the distractors will increase, facilitating the parallel detection.
In the studies of Müller et al. (2003) and Wolfe et al. (2003), the intercept of the RT $\times$ set size function was changed by manipulating top-down control. However, the slope of feature search remained flat; thus, it seems that top-down control had no effect on the parallel pattern of feature search.

However, Di Lollo, Kawahara, Zuvic, and Visser (2001; Di Lollo, Smilek, Kawahara, \& Ghorashi, 2005) reported evidence against the suggestion above. In their study, the effect of top-down modulation was measured by comparing the efficiency of feature search in different task conditions, including single- and dual-task conditions with different T1-T2 interstimulus intervals (ISIs). The singletask condition was identical to that in Sagi and Julesz's (1985) detection task, in which observers discriminated the number of horizontal or vertical target lines among diagonal background lines. In a given block of trials, the number of target lines was fixed at (1) one or two, (2) two or three, or (3) three or four. Under the dual-task condition, the observers first reported whether or not the top and bottom lines of a hexagon were tilted in the same direction (T1) and then enumerated the targets (T2). The results revealed that in the single- or dual-task condition with a long T1-T2 ISI, threshold exposure for $85 \%$ accuracy remained approximately constant as a function of target set size. However, the threshold increased sharply when $\mathrm{T} 1$ and $\mathrm{T} 2$ were presented simultaneously or the ISI between them was $100 \mathrm{msec}$.

On the basis of these results, Di Lollo et al. (2001) proposed a dynamic control theory, suggesting that the slope for feature search can be modulated by task set re-

M. Shen, mwshen@zju.edu.cn 
configuration. They argued that early visual processing is not performed by built-in feature analyzers, but by a set of input filters whose functional characteristics can be dynamically reconfigured under the control of the prefrontal cortex, so as to handle incoming stimuli with maximum efficiency in different conditions. They suggested that the key factor determining the efficiency of early visual processing is not the nature of the stimulus, but whether or not it can be handled optimally by the current configuration. According to this theory, when the T1-T2 ISI is too short for the visual system to be suitably configured, the mismatch between the information from input features and the current task set will substantially impair the efficiency of feature detection, and the search for that feature will yield steep slopes.

A flexible mechanism whose functions can be dynamically reconfigured has also been suggested by VanRullen, Reddy, and Koch (2004). However, whether or not the pattern of feature processing is actually affected by reconfiguration still needs further investigation. Here, we suggest two possible explanations for the inconsistency between the results obtained by Müller et al. (2003) and Wolfe et al. (2003) and those of Di Lollo et al. (2001) concerning the effect of top-down control on the slope of feature search.

First, the paradigms in which the top-down information was manipulated by cue validity (Müller et al., 2003) and dimension uncertainty (Wolfe et al., 2003) consisted of a single task. Despite the fact that the target-defining dimension was uncertain from trial to trial, the target was always a singleton item. It could be argued that the singleton search strategy (Bacon \& Egeth, 1994) alone was effective enough to yield a parallel detection. However, in Di Lollo et al.'s (2001) dual-task paradigm, T2 was a feature search task, whereas $\mathrm{T} 1$ was not. The effect of top-down control was measured by comparing the performance in the single-task condition with that in the dualtask condition. The sharply increased slope was attributed to the occupancy of the central resource by processing T1 and reconfiguring the task set from a non-feature-search task into a feature search task.

Alternatively, the steep slope in Di Lollo et al. (2001; Di Lollo et al., 2005) might not reflect a serial feature detection process. In their paradigm, the observers were required to determine the number of the targets. In contrast to most other studies, search slope was measured by varying target set size, rather than distractor set size. We suggest that two subprocesses are involved in determining the number of the targets: (1) individualizing the targets from the distractors, which is achieved by the mechanism of feature processing, and (2) enumerating the individualized targets through subitizing or counting (Beckwith \& Restle, 1966). In Di Lollo et al. (2001; Di Lollo et al., 2005), the effect of task set reconfiguration on each of the two subprocesses could not be separated. The increased slope might be produced by an impairment of either of the two subprocesses. It could be suggested that in Di Lollo et al., what was subjected to task set reconfiguration was the enumeration process, whereas the individualizing process remained unaffected.
The dual-task paradigm, in which two tasks are executed sequentially, has also been employed in other research on the performance of feature search (Joseph, Chun, \& Nakayama, 1997; Kawahara, Di Lollo, \& Enns, 2001). In these studies, observers identified a target letter in a rapid serial visual presentation stream (T1) and then completed a feature search task (T2). The results revealed that when the T1-T2 ISI was less than $500 \mathrm{msec}, \mathrm{T} 2$ was significantly impaired by the prior task. However, since the set size of the feature search task was not manipulated, it was not clear whether the drop in performance could be partly attributed to the increase of search slope.

The two alternative explanations discussed above regarding the results of Di Lollo et al. (2001; Di Lollo et al., 2005) were examined in the present study. Experiment 1 was a replication of Di Lollo et al.'s (2001) experiment with a different target-defining feature. Since variation in target set sizes might influence performance on both feature processing and enumeration, in the subsequent two experiments, target set size was held at a fixed level. The efficiency of feature search was measured by manipulating distractor set size.

\section{EXPERIMENT 1}

Our pilot experiments showed that searching for a target defined by orientation was facilitated, rather than impaired, as distractor set size was increased. In addition, when the targets were defined by color, no significant negative slope was found. Therefore, in the present experiment, the target was defined by color, in order to avoid the complication caused by a negative slope. The purpose of this experiment was to determine whether, when targets were defined by a feature different from that in Di Lollo et al. (2001), the same pattern of results could be obtained.

\section{Method}

Participants. Six undergraduate and postgraduate students participated in this experiment. Their ages ranged from 20 to 24 years. All had normal color vision and normal or corrected-to-normal visual acuity.

Apparatus and Stimuli. The stimuli were presented in the center of a 17-in. monitor (100-Hz refresh rate), which was controlled by a Pentium III 800 computer. The participants were tested individually in a darkened room, seated about $57 \mathrm{~cm}$ from the computer. All the stimuli were presented on a gray (CIE: $x=0.298, y=0.334 ; Y=$ $\left.14.22 \mathrm{~cd} / \mathrm{m}^{2}\right)$ background. The color of the hexagon was white $(Y=$ $55.02 \mathrm{~cd} / \mathrm{m}^{2}$ ). Its diameter was $7.5^{\circ}$, with a thickness of $0.12^{\circ}$. The top and bottom lines of the hexagon were always tilted $14^{\circ}$ away from the horizontal, clockwise or counterclockwise. This resulted in two types of hexagons: parallel and diverging. Feature search items were distributed within an imaginary circle with a $6.5^{\circ}$ diameter. The distractors were white squares $\left(Y=55.02 \mathrm{~cd} / \mathrm{m}^{2}\right)$, and the targets were red squares (CIE: $x=0.560, y=0.378 ; Y=14.64 \mathrm{~cd} / \mathrm{m}^{2}$ ). Each item constituted a $0.6^{\circ} \times 0.6^{\circ}$ visual angle. The mask for each of the search items was composed of two red and two white squares that were randomly abutted to form a $2 \times 2$ checkerboard pattern $\left(1.2^{\circ} \times 1.2^{\circ}\right.$ total size $)$.

Design and Procedure. There was a 2 (single task or dual task) $\times 3$ (target set size: one vs. two, two vs. three, or three vs. four) within-groups design. The stimuli sequence in a single trial is illustrated in Figure 1. The relevant stimulus display was presented 


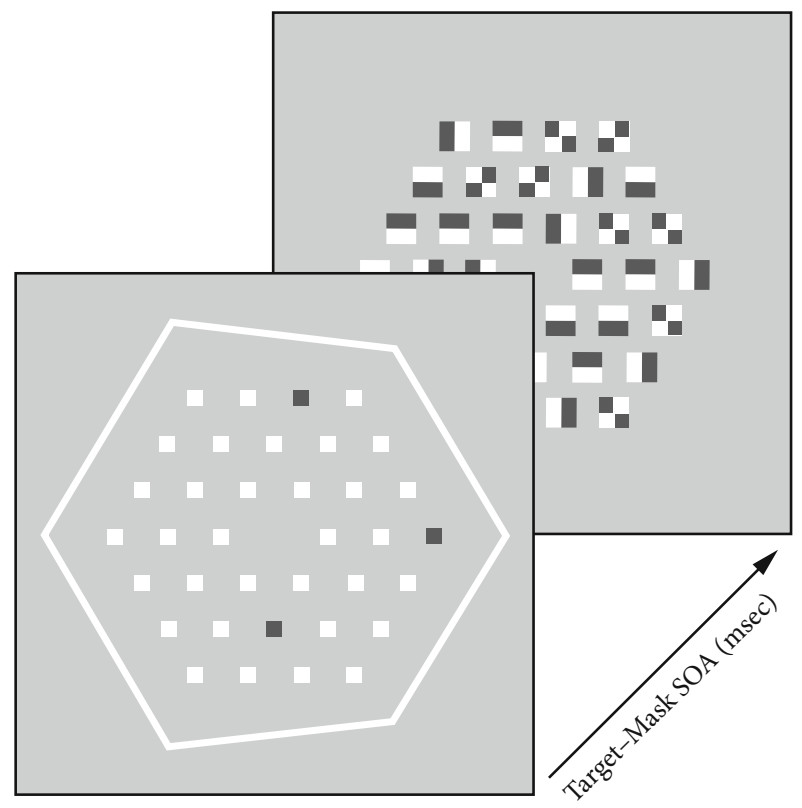

Figure 1. Schematic illustration of the experimental paradigm used in Experiment 1. The top and bottom lines of the hexagon shown here diverge. The feature search (T2) display shown here contains three red squares.

for $24 \mathrm{msec}$. The masking pattern was presented for $200 \mathrm{msec}$ at an ISI, the duration of which was under the control of the PEST staircase procedure described below. In the single-task condition, the observers were required to ignore the hexagon and to report only the number of the red squares among the white distractors (feature enumeration task). In a given block of trials, the number of red squares was fixed at (1) one or two, (2) two or three, or (3) three or four. The observers pressed one of the two keys to indicate the number of the targets. A dynamic threshold tracking procedure (PEST; Taylor \& Creelman, 1967) was used to converge on the critical target mask ISI at which the observer made approximately $85 \%$ correct responses.

In the dual-task condition, two responses were required. First, the observers pressed either the "S" or the " $F$ " key on the keyboard to indicate whether or not the top and the bottom lines of the hexagon were tilted in the same direction. Second, the observers completed the feature enumeration task, as in the single-task condition. The comparison of the top and bottom lines of the hexagon was the primary task, to which the observers were instructed to pay full attention. The PEST procedure tracked performance on the feature enumeration task. In converging to the critical ISI, PEST utilized only those trials on which the response to the primary task was correct.

A given block continued until the PEST procedure obtained the critical ISI, making the number of trials uncertain. There were approximately 250 trials in each block, for a total of up to 1,500 trials. The sequence of blocks with different combinations of task condition and target set size was counterbalanced between participants. In order to further reduce practice effects, each observer underwent 2,000 practice trials prior to the formal experiment.

\section{Results and Discussion}

The mean durations of the critical ISI, plotted as a function of target set size for each of the two task conditions, are shown in Figure 2. A repeated measure analysis revealed that the main effects of task condition $[F(1,5)=104.315$, $p<.001]$ and target set size $[F(2,10)=31.831, p<.001]$ were significant. Most important, the interaction between the two variables was also significant $[F(2,10)=15.099$, $p=.001]$, indicating that the slope of the enumeration task was significantly steeper in the dual-task condition than in the single-task condition.

In the present experiment, with a different targetdefining feature, the main finding of Di Lollo et al. (2001; Di Lollo et al., 2005) was confirmed, showing that efficiency for the feature enumeration task was, indeed, impaired by the additional task. In Experiment 2, we investigated whether such an impairment could be explained in terms of a serial feature search.

\section{EXPERIMENT 2}

In order to exclude variation in the efficiency of the enumeration process and to focus on feature processing, in the present experiment, target set size was fixed at the level of two versus three, and distractor set size was manipulated. The stimuli, procedure, and task requirements were the same as those in Experiment 1, but a different design was adopted. In Experiment 1, trials with different combinations of target set sizes and task conditions were blocked, and the dependent variable was the critical ISI at $85 \%$ accuracy. In the present experiment, the single- and dual-task conditions were still blocked, but trials with different distractor set sizes were randomly mixed in each of the two task conditions. The dependent variable was accuracy. Such a difference in design was unlikely to have any effect on the interaction between task condition and set size. For unspeeded responses, target-mask ISI and accuracy were two trade-off indexes. On the one hand, the variation in task difficulty caused by different set sizes could be reflected by the critical ISI at $85 \%$ accuracy. On the other hand, the variation in search difficulty could also be reflected by accuracy when the ISI was the same for trials with different set sizes. When distractor set sizes were

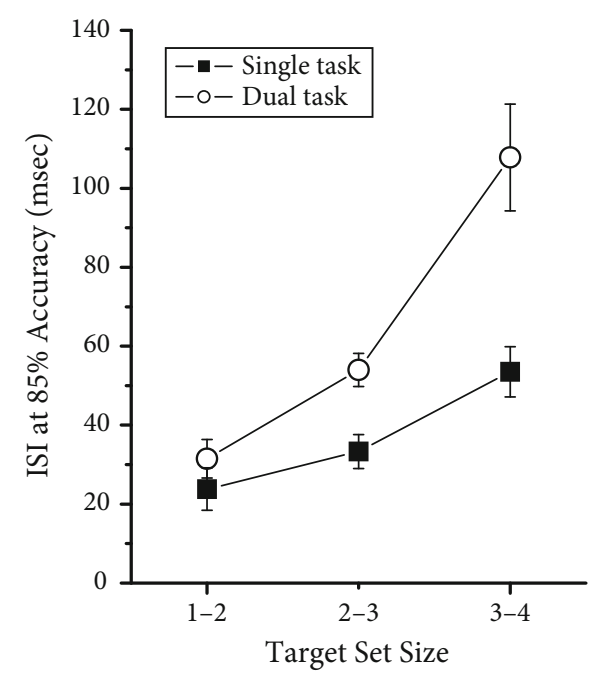

Figure 2. Results from Experiment 1: Target-Mask interstimulus interval (ISI) at $85 \%$ accuracy for the single-task and the dual-task conditions, plotted as a function of target set size. Error bars represent the average standard error. 


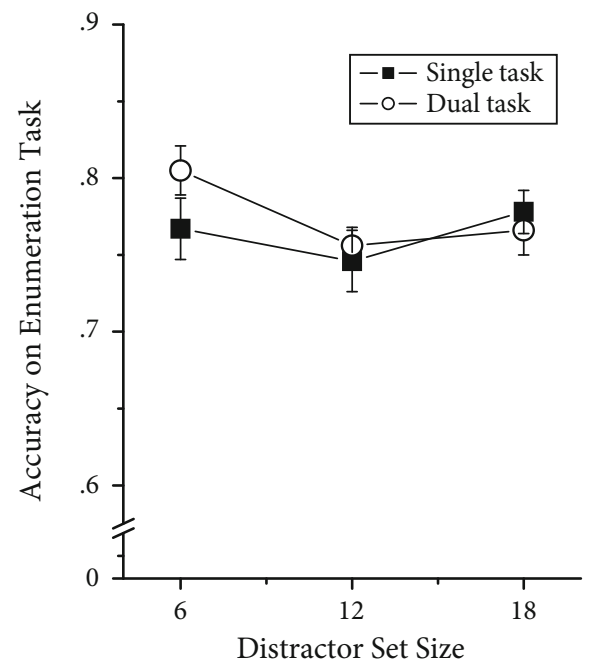

Figure 3. Results from Experiment 2: Mean accuracy on the enumeration task for the single-task and the dual-task conditions, plotted as a function of distractor set size. Error bars represent the average standard error.

mixed, the number of trials required was substantially decreased (see the design described in detail in the Design and Procedure section), and any potential contamination caused by the sequence of different set sizes could also be avoided.

If the steep slope in the dual-task condition in Experiment 1 could be attributed to the drastic drop in the efficiency of feature detection, then in the present study, when distractor set size was manipulated, the feature enumeration task should also yield a much steeper slope in the dual-task condition. However, if task set reconfiguration has no effect on the efficiency of individualizing targets from distractors and the steep slope in Experiment 1 was produced exclusively by the enumeration process, there should be no interaction between task condition and distractor set size.

\section{Method}

Participants. Twelve undergraduate and postgraduate students participated in this experiment. Their ages ranged from 20 to 24 years. All had normal color vision and normal or corrected-tonormal visual acuity.

Apparatus and Stimuli. The apparatus and stimuli were identical to those in Experiment 1.

Design and Procedure. There was a 2 (single task or dual task) $\times$ 3 (distractor set size: 6,12, or 18) within-group design. Each cell of the factional design consisted of 40 trials, for a total of up to 240 trials. The task conditions were blocked. Within each block, trials with different distractor set sizes were randomly mixed. Target set size was fixed at the level of two versus three. There were 80 practice trials before each block. The sequence of task conditions was counterbalanced between participants. The stimuli sequence for a single trial and the task requirements in the single- and dual-task conditions were identical to those in Experiment 1. In the experiment session, the duration of the ISI was dynamically varied, so that mean accuracy for the enumeration task in different task conditions could be maintained in the same measurable range. For each observer, accuracy of feature enumeration, averaged over the three distractor set sizes, was calculated automatically every 12 trials. If it was below $75 \%$, the duration was prolonged by $12 \mathrm{msec}$; if it was above $83.3 \%$, it was decreased by $12 \mathrm{msec}$.

\section{Results and Discussion}

Average accuracy for the primary task was $92.2 \%$. Trials in which the response to this task was wrong were eliminated from further analysis. The mean ISI was $34 \mathrm{msec}$ in the single-task condition and $62 \mathrm{msec}$ in the dual-task condition. An ANOVA showed that the difference between them was significant $[F(1,11)=11.398, p<.01]$, indicating that with the additional task, the overall performance of the feature enumeration task was impaired. To achieve a comparable accuracy, a longer ISI was required in the dual-task condition than in the single-task condition.

Figure 3 shows the observers' accuracy on the feature enumeration task plotted as a function of distractor set size for each of the two task conditions. A repeated measure analysis revealed no significant effect of the task condition $[F(1,11)=1.128, p>.1]$, suggesting that the dynamic tracking procedure was effective in keeping mean accuracy at the same level in both the single- and the dual-task conditions. The main effects of distractor set size were not significant $[F(2,22)=1.853, p>.1]$. Of most interest, there was no significant interaction between task condition and distractor set size $[F(2,22)=0.978$, $p>1$ ], indicating that task set reconfiguration did not yield a steeper slope in feature search.

The present results were consistent with the findings in Müller et al. (2003) and Wolfe et al. (2003) but were inconsistent with the prediction of dynamical control theory. It could be suggested that the much steeper slope for the feature enumeration task in Experiment 1 should be attributed to the enumeration process, rather than to the feature detection process.

In the next experiment, we further investigated whether task set reconfiguration can change the slope of feature search when it is guided primarily by top-down information.

\section{EXPERIMENT 3}

According to Wolfe's guided search theory, attention is guided by the sum of top-down activation and bottomup activation. Top-down activation is controlled by the observer's task set. Bottom-up activation is based on comparisons of each item with its neighbors. Since homogenous distractors were displayed in Experiment 2, it could be argued that bottom-up activation alone could have made the targets more highly activated than any of the distractors, resulting in a parallel feature search whether or not top-down control was suitably established. It is therefore possible that the slope of feature search would be increased by task set reconfiguration when no difference existed between the bottom-up activations of the targets and the distractors and individualizing the targets could be accomplished only by top-down guidance. In the present experiment, the difference in bottom-up activation was minimized by extending the color set of 
the distractors from one color to six colors. In the feature search array, the number of target items was greater than or equal to the number of distractors. According to the guided search model, in such conditions, the bottom-up activation of the target should not be higher than that for any of the distractors, and top-down guidance should play a dominating role.

Heterogeneous distractors can increase the difficulty of feature search, so even in the single-task condition, feature search might yield a slight search slope. However, just as in the two experiments above, the primary aim of the present experiment was to find out whether there was any interaction between task condition and set size.

\begin{abstract}
Method
Participants. Twelve undergraduate and postgraduate students participated in this experiment. Their ages ranged from 21 to 25 years. All had normal color vision and normal or corrected-tonormal visual acuity.

Stimuli, Design, and Procedure. The colors of the distractors were selected from a set of six colors: blue (CIE: $x=0.149, y=$ $0.091 ; Y=8.37 \mathrm{~cd} / \mathrm{m}^{2}$ ), green (CIE: $x=0.267, y=0.637 ; Y=$ $\left.52.82 \mathrm{~cd} / \mathrm{m}^{2}\right)$, yellow (CIE: $\left.x=0.417, y=0.485 ; Y=50.1 \mathrm{~cd} / \mathrm{m}^{2}\right)$, violet (CIE: $\left.x=0.267, y=0.126 ; Y=16.47 \mathrm{~cd} / \mathrm{m}^{2}\right)$, cyan (CIE: $x=$ $\left.0.217, y=0.271 ; Y=45.09 \mathrm{~cd} / \mathrm{m}^{2}\right)$, and black $\left(Y=1.339 \mathrm{~cd} / \mathrm{m}^{2}\right)$. In each of the conditions for the three distractor set sizes $(6,12$, or 18 ), the number of distractors with each of the six colors was identical. The mask for each of the feature search items was composed of four colored squares that were abutted to form a $2 \times 2$ checkerboard pattern $\left(1.20^{\circ} \times 1.20^{\circ}\right.$ total size $)$. The colors of the squares in each mask were randomly selected from the color set of the distractors. Since feature search in the present experiment was much more difficult than that in Experiment 2, the duration of the relevant stimuli (including the hexagon and feature search array) was prolonged to $84 \mathrm{msec}$. All of the other aspects of the stimuli, experimental design, and procedure were identical to those in Experiment 2.
\end{abstract}

\section{Results and Discussion}

Average accuracy for the primary task was $92.4 \%$. Trials on which response to the primary task was wrong were eliminated from further analysis. The mean ISI in the single-task condition was $26 \mathrm{msec}$, and it was $86 \mathrm{msec}$ in the dual-task condition. An ANOVA revealed a significant difference $[F(1,11)=9.890, p<.01]$, indicating that task set configuration did impair the overall performance of the feature enumeration task. To achieve comparable accuracy, a longer ISI was required in the dual-task condition than in the single-task condition.

Figure 4 shows the observers' accuracy on the feature enumeration task, plotted as a function of distractor set size for each of the two task conditions. A repeated measure analysis revealed no significant effect of task condition $[F(1,11)=1.508, p>.1]$, indicating that the dynamictracking procedure was effective in keeping mean accuracy at the same level in both the single- and the dual-task conditions. The main effects of distractor set size were significant $[F(2,22)=42.505, p<.001]$, showing that with heterogenous distractors, feature search was not parallel. Such a nonparallel feature search could be explained by several theories of visual search (Duncan \& Humphreys, 1989; Treisman \& Gormican, 1988; Wolfe, 1994). However, the primary interest of the present experiment was

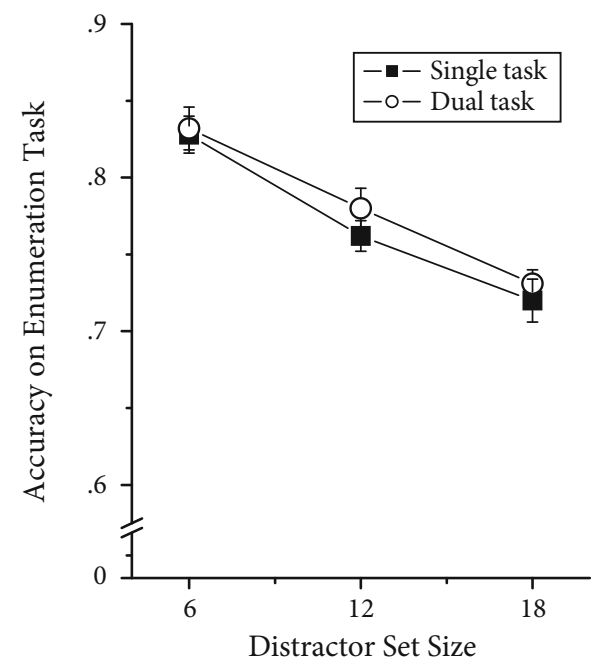

Figure 4. Results from Experiment 3: Mean accuracy on the enumeration task for the single-task and the dual-task conditions, plotted as a function of distractor set size. Error bars represent the average standard error.

the interaction between task condition and distractor set size, which was not significant $[F(2,22)=0.133, p>.1]$. This indicates that even when feature search was guided by top-down information, the task set reconfiguration still did not impair the efficiency of feature search.

However, the present results were not necessarily inconsistent with dynamic control theory. Since the main effects of distractor set size were significant, it could be argued that feature search with heterogenous distractors was actually carried out with a quite low efficiency. According to dynamical control theory, for a low-efficiency search task, the target feature cannot be suitably matched even in the single-task condition; thus, during task set reconfiguration, the task is performed just as inefficiently. Di Lollo et al. (2005) did report that task set reconfiguration could not further increase the steep slope yielded by searching for a rotated $\mathrm{T}$ among rotated Ls. This alternative explanation was examined in Experiment 4.

\section{EXPERIMENT 4}

In this experiment, the observers were required to make a speeded response to a feature search task that was identical to that in Experiment 3. By comparing its RT $\times$ set size functions with those for the various visual search tasks reviewed in Wolfe (1998), we can decide whether the task can be classified as an efficient search task.

\section{Method}

Participants. Six undergraduate students participated in this experiment. Their ages ranged from 19 to 22 years. All had normal color vision and normal or corrected-to-normal visual acuity.

Stimuli, Design, and Procedure. All aspects of this experiment were identical to those of the single-task condition in Experiment 3, with the exception that the mask for each of the feature search items was removed. The stimuli remained on the screen until a response was initiated. 


\section{Results and Discussion}

Figure 5 shows the observers' RTs for the feature enumeration task, plotted as a function of distractor set size. The main effects of distractor set size were significant $[F(2,10)=8.768, p<.01]$. However, a linear regression showed that the slope of the RT $\times$ set size function was only $2.2 \mathrm{msec} /$ item, which was much smaller than the slope for the $\mathrm{T}$ and $\mathrm{L}$ discrimination task. This small search slope indicated that the feature search conducted in Experiment 3 can still be classified as a high-efficiency search (Wolfe, 1998), suggesting that the results of Experiment 3 cannot be explained by dynamic control theory.

\section{GENERAL DISCUSSION}

According to dynamic control theory, perceptions emerge when the stimulus input is matched to a multidimensional template stored within the system. When a suitable target template is embodied in the input filter's configuration, the stimulus matching this template will be perceived directly and immediately during the feedforward process. Searching for such a target will be efficient. When the stimulus is not configurable, or when there has not been sufficient time for the system to be suitably configured, template matching requires reentrant processing, in which perceptions emerge from interactive exchanges between brain regions linked by reentrant pathways. The most important view of this theory is that if the task set is not suitably configured, even the processing of basic features will yield a steep slope.

This prediction was carefully reexamined in the present research by dividing the feature enumeration task adopted in Di Lollo et al. (2001; Di Lollo et al., 2005) into two subprocesses: (1) the target detection process and (2) the enumeration process. In order to exclude variation in the efficiency of the enumeration process, target set size was fixed, and distractor set size was manipulated. The results revealed that whether search was guided by bottom-up information or by top-down information, the slope of feature search was not affected by an additional task.

In contradiction to dynamic control theory, these findings indicate that the "built-in" feature analyzer hypothesis, accounting for the high efficiency of feature search, is still plausible. Feature integration theory (FIT; Treisman, 1998; Treisman \& Gormican, 1988) suggests that the basic features of the whole stimulus array are represented in parallel in separated feature maps. Whether a specific feature is presented can be resolved by simply checking the pooled activation of the relevant feature map. According to this theory, what can be dynamically adjusted are the priorities of multiple independent feature maps in the checking process. The feature map that matches the current task set best gains the highest priority and can be checked first. When the target is not detected in that map, multiple maps will be sequentially checked, according to their priorities. When the task set is unsuitable, such a process of sequential checking will prolong the overall duration of feature search. However, the pattern of feature search will not be changed, since in each of these maps, basic features are still represented in parallel.

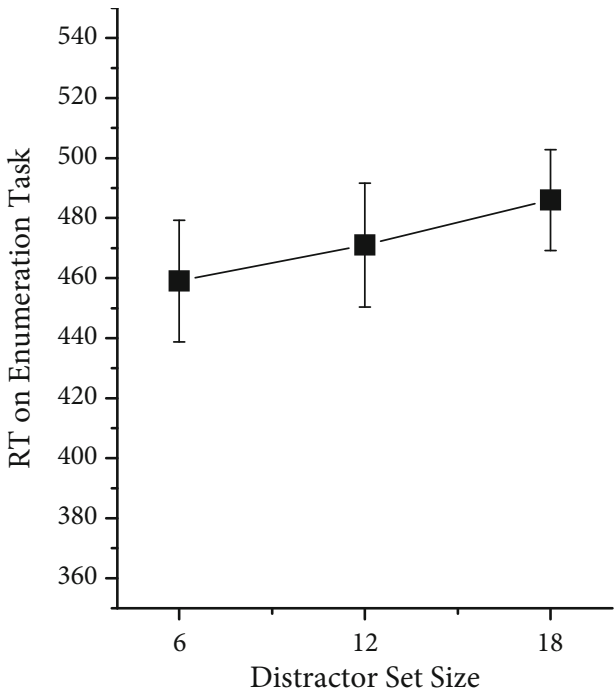

Figure 5. Results from Experiment 4: Mean reaction times (RTs) on the enumeration task, plotted as a function of distractor set size. Error bars represent the average standard error.

Although consistent with the present results, the assumption that feature detection is achieved by checking the activation of separated feature maps has been challenged by many other findings. There is evidence showing that targets defined by complicated properties (Enns \& Rensink, 1991; He \& Nakayama, 1992; Wolfe, Cave, \& Franzel, 1989; Wolfe, Friedman-Hill, \& Bilsky, 1994) can also be searched in parallel. Since it is unlikely that there are so many feature analyzers, each specified to one of these complicated properties, Nakayama and Joseph (1998) suggested that parallel processing is based on the analysis of abstract and generalized information of the whole visual scene, rather than on checking the activation of some specific feature maps.

One influential modification of FIT is guided search theory (Wolfe, 1994). According to this theory, visual attention is guided by the output of an activation map in which the activations of all the separate feature maps are summed up. Taking the activation of the target as the signal and the activation of the distractors as the distracting noise, when the signal/noise $(\mathrm{S} / \mathrm{N})$ ratio is much greater than 1 , attention will almost always be deployed to the target first. Since the serial attentive processing of the distractors can be avoided, a flat slope for the RT $\times$ set size function can be obtained. Wolfe et al. (2003) further proposed that besides the rank order of the target on an activation list of all items, performance is also influenced by the salience of the difference between the target and the distractors. The greater the $\mathrm{S} / \mathrm{N}$ ratio, the more quickly information can accumulate. The more quickly it accumulates, the more quickly a decision can be made about the identity of the item. They also suggest what can be dynamically modulated are the weights of multiple feature dimensions in the activation map. In natural conditions, suitable top-down control can give as much weight as possible to the target feature dimension, so that the $\mathrm{S} / \mathrm{N}$ ratio 
can be optimized. When the task set is not suitably configured, the weight of the target feature dimension is weakened, resulting in a decreased $\mathrm{S} / \mathrm{N}$ ratio; thus, detection of the target will be slowed. However, if the decreased S/N ratio is still significantly greater than 1 , attention can still be directly deployed to the target, so the high efficiency of feature search will be maintained.

This theory can easily explain the results of Experiment 2 , in which even when the task set was not suitably configured, the contrast between the target and the homogenous distractors could produce an $\mathrm{S} / \mathrm{N}$ ratio significantly greater than 1 . So an additional task cannot increase the slope of feature detection. However, for a feature search with heterogeneous distractors, as in Experiments 3 and 4, the significant main effects of distractor set size showed that despite the fact that the $\mathrm{S} / \mathrm{N}$ ratio was still somewhat greater than 1 , the target was not always the item of highest activation. In some trials, distractors might be attentively processed. With such an $\mathrm{S} / \mathrm{N}$ ratio, when the salience of the targets was decreased by the unsuitable top-down control, their rank orders on the list of activation of all items should also be lowered. However, the results of Experiment 3 showed that the slope of feature search was unchanged in the dual-task condition, which was inconsistent with the prediction above.

On the basis of the results of the present study, it can be assumed that the visual system establishes a suitable task set first and then executes the search task with optimal efficiency. This does not support the theory of Di Lollo et al. (2001). We suggest that during a low-efficiency search, focal attention quickly shifts among items (usually less than $50 \mathrm{msec} /$ item), requiring the visual system to make an immediate decision about which one of the sequentially processed items is the target. To achieve such a dynamic discrimination, it is necessary to keep information about the target online in working memory, which is functionally identical to establishing a suitable task set (Downing, 2000; Soto, Heinke, Humphreys, \& Blanco, 2005). In the dual-task condition, when the information about the target is offline because of the execution of a competing task, immediate identification of the target among sequentially processed items cannot be carried out. Once information about the target is activated in working memory, the salience of the target in the activation map will also be automatically increased. Thus, the search can be executed just as efficiently as in the single-task condition.

Since there was no interaction between task condition and distractor set size, the much steeper slope for the feature enumeration task in Experiment 1 and in Di Lollo et al. (2001) can be attributed to the low efficiency of the enumeration process. Previous studies have shown that the visual system can enumerate up to four items in a parallel fashion. However, when there are more than four items, the RT, or the time required for accurate counting, steeply increases with the increasing number of items. The fast and efficient enumeration of small numbers of items is called subitization (Trick \& Pylyshyn, 1993, 1994). According to Trick and Pylyshyn, there are a limited number of "fingers" of instantiation (FINSTs) that can be used to define individual items in the visual field. Subitization arises because a small number of items can be enumerated by assigning FINSTs to them in parallel and then associating the number of bound FINSTs directly with number names.

According to this theory, what is distracted by task set reconfiguration is the parallel subitization mechanism. One possible explanation is that task set reconfiguration is a progressive process. When optimal top-down control has been established for individualizing targets from distractors, it still lasts for a while, during which time subitization is simultaneously carried out. However, before the cognitive resource assigned to the additional task is completely released, the capacity of subitization is reduced. This results in a steep slope for counting even when the number of targets never exceeds four. We hypothesize that the number of "fingers" is not fixed at four, but is dependent on the availability of the central resource. Since the occupancy of the central resource by task set reconfiguration decreases the number of "fingers," the subitizing range shrinks accordingly.

\section{AUTHOR NOTE}

This research was supported by the National Natural Science Foundation of China (No. 30570604), the Fund of the Chinese Ministry of Education for Doctoral Programs in Universities (No. 20030335024), and the Research Center of Language and Cognition, Zhejiang University. We thank Jeremy Wolfe, Vincent Di Lollo, Anne Treisman, and two anonymous reviewers for their helpful comments on various drafts of the article. Correspondence concerning this article should be addressed to M. Shen, Department of Psychology, Xixi Campus, Zhejiang University, Hangzhou, 310028, People's Republic of China (e-mail: mwshen@zju .edu.cn).

\section{REFERENCES}

Bacon, W. F., \& Egeth, H. E. (1994). Overriding stimulus-driven attentional capture. Perception \& Psychophysics, 55, 485-496.

Beckwith, M., \& Restle, F. (1966). Process of enumeration. Psychological Review, 73, 437-444.

Di Lollo, V., Kawahara, J.-I., Zuvic, S. M., \& Visser, T. A. W. (2001). The preattentive emperor has no clothes: A dynamic redressing. Journal of Experimental Psychology: General, 130, 479-492.

Di Lollo, V., Smilek, D., Kawahara, J.-I, \& Ghorashi, S. M. S. (2005). System reconfiguration, not resource depletion, determines the efficiency of visual search. Perception \& Psychophysics, 67, 1080-1087.

DownING, P. E. (2000). Interactions between visual working memory and selective attention. Psychological Science, 11, 467-473.

Duncan, J., \& Humphreys, G. W. (1989). Visual search and stimulus similarity. Psychological Review, 96, 433-458.

Enns, J. T., \& Rensink, R. A. (1991). Preattentive recovery of threedimensional orientation from line drawings. Psychological Review, 98, 335-351.

He, Z. J., \& NaKayama, K. (1992). Surfaces versus features in visual search. Nature, 359, 231-233.

Hochstein, S., \& Ahissar, M. (2002). View from the top: Hierarchies and reverse hierarchies in the visual system. Neuron, 36, 791-804.

Joseph, J. S., Chun, M. M., \& Nakayama, K. (1997). Attentional requirements in a "preattentive" feature search task. Nature, 387, 805-807.

Kawahara, J.-I., Di Lollo, V., \& EnNs, J. T. (2001). Attentional requirements in visual detection and identification: Evidence from the attentional blink. Journal of Experimental Psychology: Human Perception \& Performance, 27, 969-984.

Müller, H. J., Reimann, B., \& Krummenacher, J. (2003). Visual search for singleton feature targets across dimensions: Stimulus- and expectancy-driven effects in dimensional weighting. Journal of Experimental Psychology: Human Perception \& Performance, 29, 1021-1035. 
Nakayama, K., \& Joseph, J. S. (1998). Attention, pattern recognition, and pop-out visual search. In R. Parasuraman (Ed.), The attentive brain (pp. 279-298). Cambridge, MA: MIT Press.

SAGi, D., \& Julesz, B. (1985). "Where" and "what" in vision. Science, 228, 1217-1219.

Soto, D., Heinke, D., Humphreys, G. W., \& Blanco, M. J. (2005). Early, involuntary top-down guidance of attention from working memory. Journal of Experimental Psychology: Human Perception \& Performance, 31, 248-261.

TAYlor, M. M., \& Creelman, C. D. (1967). PEST: Efficient estimates on probability functions. Journal of the Acoustical Society of America, 41, 782-787.

Treisman, A. [M.] (1998). Feature binding, attention and object perception. Proceedings of the Royal Society of London: Series B, 353, 1295-1306.

Treisman, A. M., \& Gelade, G. (1980). A feature-integration theory of attention. Cognitive Psychology, 12, 97-136.

Treisman, A. [M.], \& Gormican, S. (1988). Feature analysis in early vision: Evidence from search asymmetries. Psychological Review, 95, 15-48.

Trick, L. M., \& Pylyshyn, Z. W. (1993). What enumeration studies can show us about spatial attention: Evidence for limited capacity preattentive processing. Journal of Experimental Psychology: Human Perception \& Performance, 19, 331-351.
TRICK, L. M., \& PYlYSHYN, Z. W. (1994). Why are small and large numbers enumerated differently? A limited-capacity preattentive stage in vision. Psychological Review, 101, 80-102.

VanRullen, R., Reddy, L., \& Koch, C. (2004). Visual search and dual tasks reveal two distinct attentional resources. Journal of Cognitive Neuroscience, 16, 4-14.

Wolfe, J. M. (1994). Guided Search 2.0: A revised model of visual search. Psychonomic Bulletin \& Review, 1, 202-238.

Wolfe, J. M. (1998). What can 1 million trials tell us about visual search? Psychological Science, 9, 33-39.

Wolfe, J. M., Butcher, S. J., Lee, C., \& Hyle, M. (2003). Changing your mind: On the contributions of top-down and bottom-up guidance in visual search for feature singletons. Journal of Experimental Psychology: Human Perception \& Performance, 29, 483-502.

Wolfe, J. M., Cave, K. R., \& Franzel, S. L. (1989). Guided search: An alternative to the feature integration model for visual search. Journal of Experimental Psychology: Human Perception \& Performance, 15 419-433.

Wolfe, J. M., Friedman-Hill, S. R., \& Bilsky, A. B. (1994). Parallel processing of part-whole information in visual search tasks. Perception \& Psychophysics, 55, 537-550.

(Manuscript received February 15, 2005; revision accepted for publication April 7, 2006. 